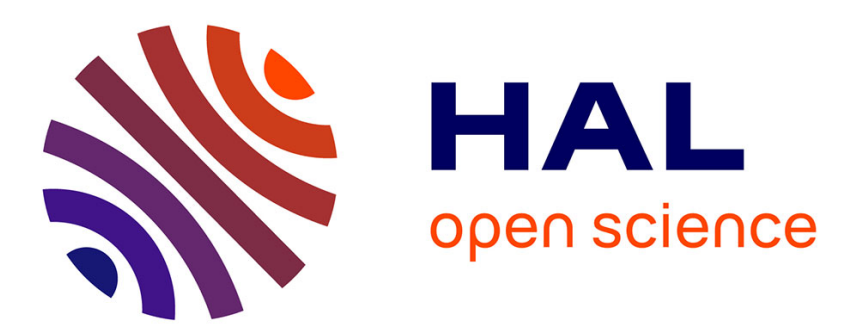

\title{
Un modèle associatif de circuits courts de proximité pour les épiceries sociales et solidaires: vers une démocratie alimentaire?
}

Dominique Paturel, Aurélie Carimentrand

\section{- To cite this version:}

Dominique Paturel, Aurélie Carimentrand. Un modèle associatif de circuits courts de proximité pour les épiceries sociales et solidaires: vers une démocratie alimentaire?. RIODD 2016, Jul 2016, SaintÉtienne, France. 10.3917/ror.131.0043 . hal-01349973

\section{HAL Id: hal-01349973 \\ https://hal.science/hal-01349973}

Submitted on 29 Jul 2016

HAL is a multi-disciplinary open access archive for the deposit and dissemination of scientific research documents, whether they are published or not. The documents may come from teaching and research institutions in France or abroad, or from public or private research centers.
L'archive ouverte pluridisciplinaire HAL, est destinée au dépôt et à la diffusion de documents scientifiques de niveau recherche, publiés ou non, émanant des établissements d'enseignement et de recherche français ou étrangers, des laboratoires publics ou privés. 


\title{
Un modèle associatif de circuits courts de proximité pour les épiceries sociales et solidaires : vers une démocratie alimentaire?
}

\section{Dominique PATUREL}

INRA

UMR 951 Innovation

2 place Viala 34060 Montpellier cedex 02

Téléphone: +33 (0)6 65719487

dpaturel@supagro.inra.fr

\section{Aurélie CARIMENTRAND}

Maître de conférences,IUT Bordeaux Montaigne

UMR PASSAGES

12 esplanade des Antilles 33607 Pessac cedex

Téléphone : + 33 (0)5 57122135

aurelie.carimentrand@iut.u-bordeaux-montaigne.fr

\section{Résumé}

Les initiatives relevant de la construction de systèmes agroalimentaires alternatifs se multiplient. Parmi ces initiatives émergent de nouvelles modalités qui visent à rendre plus accessibles ces alternatives, y compris au niveau des acteurs de l'aide alimentaire. Le programme Uniterres mis en place au sein de l'ANDES, une des structures nationales qui regroupent les épiceries sociales et solidaires, et un des opérateurs du dispositif d'aide alimentaire français, a pour objectif d'améliorer la qualité nutritionnelle des produits proposés aux bénéficiaires des épiceries sociales. Pour cela, l'ANDES achète une partie de la production de maraîchers en situation de fragilité dans le but de soutenir un modèle d'agriculture paysanne.Dans cette communication nous interrogeons ce modèle au regard du concept de démocratie alimentaire en montrant ses leviers et ses limites.

Mots-clés : aide alimentaire, démocratie alimentaire, circuits courts, épicerie sociale et solidaire, accès à l'alimentation.

\begin{abstract}
A not-for-profit model using short food supply chains for social groceries: a possible food democracy model?

Alternative food systems are growing in number and raising academic concerns about food citizenship and food democracy. Several initiatives are working on accessibility, including food aid distribution systems. The Uniterres program, led by ANDES, a french federation of social groceries, aims to improve the nutritional value of the products they deliver to their beneficiaries. By purchasing products from smallscale local producers facing problems they also support small farmers. Our communication questions this new short food supply chain using the concept of a 'food democracy'.
\end{abstract}

Key-words: food aid, food democracy, short food supply chain, social groceries, food access. 


\title{
Un modèle associatif de circuits courts de proximité pour les épiceries sociales et solidaires : vers une démocratie alimentaire ?
}

\begin{abstract}
Résumé :Les initiatives relevant de la construction de systèmes agroalimentaires alternatifs se multiplient. Parmi ces initiatives émergent de nouvelles modalités qui visent à rendre plus accessibles ces alternatives, y compris au niveau des acteurs de l'aide alimentaire. Le programme Uniterres mis en place au sein de l'ANDES, une des structures nationales qui regroupent les épiceries sociales et solidaires, et un des opérateurs du dispositif d'aide alimentaire français, a pour objectif d'améliorer la qualité nutritionnelle des produits proposés aux bénéficiaires des épiceries sociales. Pour cela, l'ANDES achète une partie de la production de maraîchers en situation de fragilité dans le but de soutenir un modèle d'agriculture paysanne. Dans cette communication nous interrogeons ce modèle au regard du concept de démocratie alimentaire en montrant ses leviers et ses limites.
\end{abstract}

\section{Introduction}

Parmi les initiatives relevant de la construction de systèmes agroalimentaires alternatifs émergent de nouvelles modalités visant à les rendre plus accessibles. On peut citerpar exemple les paniers solidaires au sein des Associations pour le Maintien de l'Agriculture Paysanne (AMAPs) ou bien encore les chantiers d'insertion de récupération mis en place sur les marchés d'intérêt national (M.I.N).Les filières de l'aide alimentaire, actuellement en pleine reconfiguration, participent de cette dynamique. Le programme Uniterres mis en place au sein de l'Association Nationale de Développement des Épiceries Solidaires(ANDES) depuis 2012constitue actuellement le modèle de plus diffusé parmi les différentes initiatives menée en France pour connecter l'aide alimentaire avec l'agriculture locale. Mais au-delà de l'accessibilité des bénéficiaires de l'aide alimentaire à des fruits et légumes frais et du nouveau segment de marché proposé aux maraîchers locaux, le programme Uniterres participe-t-il de la construction d'un système alimentaire démocratique ? L'objet de cette communication vise justement à apporter des éléments de réponse à la problématique de la démocratie alimentaire dans la construction des circuits courts pour l'aide alimentaire en 
France. Pour ce faire nous nous appuyons sur les résultats issus du programme de recherche interventionnelle ECOALES qui a réunit les acteurs du programme Uniterres et une équipe pluridisciplinaire de chercheurs en sciences humaines et sociales ${ }^{1}$. Nous avons pour notre part mené un ensemble d'entretiens et d'enquêtes par questionnaire auprès des différents acteurs du projet, notamment auprès des agriculteurs impliqués dans Uniterres. Dans la première partie de cette communication nous présentons le modèle socio-économique de «circuits courts solidaires » caractéristique d'Uniterres en le contextualisant via la problématique de la précarité alimentaired'une part, et via les évolutions récentes au niveau des politiques publiques de l'aide alimentaire en Europe d'autre part. Dans la seconde partie nous présentons notre analyse d'Uniterres à travers le prisme de la démocratie alimentaire.

\section{1 : Approvisionner les épiceries sociales et solidaires en fruits et légumes locaux : un nouveau modèle socio-économique?}

Dans cette partie nous rappelons les principes de fonctionnement des épiceries, en rupture avec le modèle classique de l'aide alimentaire, puis nous présentons les spécificités du modèle Uniterres en termes organisationnels et économiques.

\section{1 : Précarité alimentaire et aide alimentaire}

En France, ce ne sont pas les mêmes communautés de recherche qui utilisent la notion d'insécurité alimentaire et de précarité alimentaire (Paturel et al, 2015). Tout d'abord l'insécurité alimentaire est employée en contrepoint de sécurité alimentaire : pour le dire rapidement, l'insécurité est tout ce qui manque à ce qui est requis dans la définition de la sécurité alimentaire. Cette définition s'appuie sur une conception productiviste du système

\footnotetext{
${ }^{1}$ Ce projet de recherche interventionnelle a été financé par le Fonds Français pour l'Alimentation ainsi que par la
} fondation Daniel et Nina Carasso. 
alimentaire. En choisissant la terminologie de précarité alimentaire, nous nous affilions pour notre part au courant sociologique initié par Paugam (1991, 2008)qui va mettre au cœur de la question les liens sociaux et la disqualification sociale. Cette notion de précarité alimentaire n'est pas synonyme d'insécurité alimentaire et nous met en décalage par rapport aux communautés de recherche anglophones (en nous mettant par ailleurs dans la difficulté de la traduction de celle-ci). Pour autant, notre naïveté ne nous pousse pas à croire que l'emploi banalisé de «précarité alimentaire » tel que nous le retrouvons, en France, dans le discours des acteurs de politiques publiques, des opérateurs de l'aide alimentaire ou des chercheurs préoccupés de cette question, soit aujourd'hui aussi clairement dans les traces de Paugam.

\subsubsection{La précarité alimentaire en France}

La Direction Générale de l'alimentation en 2013 a évalué le nombre de bénéficiaires de l'aide alimentaire à 3,9 millions de personnes. Il existe un problème de précarité alimentaire et celle-ci n'est pas générée par un manque de production ni par l'insuffisance de réseaux de distribution mais bien par l'insuffisance de revenus d'une partie de sa population.Une enquête du Credoc ${ }^{2}$ montre qu'un quart des ménages français estime disposer de moins de 250 euros pour finir le mois, une fois acquittées les dépenses fixes du foyer (ou « reste à vivre »). Cette proportion grimpe à 56\% pour les ménages pauvres. Parmi ces ménages pauvres, 15\% déclarent des montants négatifs, ce qui signifie qu'ils s'endettent un peu plus chaque mois. Plus de la moitié se restreignent sur l'alimentation et près du quart sur les soins médicaux afin de pouvoir continuer à payer les charges de loyer, d'énergie et autres charges courantes. Une analyse du Secours Catholique $(2010)^{3}$ sur le budget de mille familles fait état du fait que les

\footnotetext{
${ }^{2}$ Angotti et al, 2008.

${ }^{3}$ Le Secours Catholique n'a pas repris une analyse structurée de cette façon dans ses rapports récents.
} 
dépenses contraintes (loyer, énergie, transport) représentent $2 / 3$ des revenus de ces ménages. Si on ajoute les dépenses d'alimentation et d'habillement, même réduites au minimum, on dépasse le montant total du revenu. Les autres dépenses (santé, équipement du logement) ne peuvent être financées que par l'emprunt ou le découvert bancaire. D'autre part, 22,3\% des ménages agricoles sont en dessous du seuil de pauvreté, 40\% en dessous du SMIC (MSA, 2015)et leur alimentation est souvent de qualité moyenne.Les restrictions dans le poste « alimentation » vont se concrétiser par la diminution de leur consommation de viande, de poisson, de fruits et de légumes, ou encore à sauter des repas. La structure des achats va se reporter sur les féculents, plus de lait mais moins de produits laitiers transformés et des produits sucrés. L'impact sur la santé est réel et les problèmes de santé liés au surpoids, voire d'obésité ainsi que la qualité nutritionnelle dans les maladies chroniques en sont autant d'indices (Bellin-Etienne et al, 2007).

\subsubsection{L'évolution des dispositifs d'aide alimentaire vers des actions au long court et plus} participatives

Jusqu'à l'adoption de la loi no 2010-874 de modernisation de l'agriculture et de lapêche (LMAP), il n'existait pas en droit français de définition de l'aide alimentaire. A présent, le code rural et de la pêche ${ }^{4}$ précise que «l'aide alimentaire a pour objet la fourniture de denrées alimentaires aux personnes les plus démunies. Cette aide est apportée tant par l'Union européenne que par l'Etat ou toute autre personne morale ».Si cela signifie l'affirmation d'une connexion entre le système de production agricole et l'aide alimentaire (ce qui était implicite jusqu'à maintenant), on verra plus loin qu'il s'agit plutôt de confirmer l'aide alimentaire comme variable d'ajustement de la production agricole.

\footnotetext{
${ }^{4}$ Article L230-6 du code rural et de la pêche maritime créé par la loi n²010-874 de modernisation de l'agriculture et de la pêche.
} 
Dans les années 80, une augmentation importante du nombre de personnes qui sollicite une aide alimentaire oblige à repenser le dispositif de distribution. Ce qui devait être passager devient en réalité structurel ; plusieurs associations caritatives se réunissent pour créer, selon le modèle des Food Banks américaines, la première Banque Alimentaire française à Arcueil (banlieue parisienne) en 1984. L'aide alimentaire y est distribuée sous forme de colis alimentaires ou sous forme de repas.Puis en décembre 1985, Coluche crée les « Restos du Cœur » et c'est cette association qui va faire évoluer le système, en proposant un modèle qui va centraliser les achats pour avoir un poids dans la négociation des prix. Ce sera également Coluche qui impulsera la loi dont un amendement porte son nom ${ }^{5}$, permettant de bénéficier d'une réduction d'impôt dès lors qu'un don est fait à une association dont l'objet est de lutter contre la pauvreté : ce qui est le cas des associations d'aide alimentaire. Mais de nombreuses actions locales existent, portées par les centres communaux d'action sociale (CCAS). Ils s'inscrivent depuis de longues décennies sur l'intervention d'urgence et sur une aide complémentaire pour certaines catégories de population comme les personnes âgées. La distribution se fait sous forme de colis alimentaires mais également de bons qui s'échangent dans les commerces de grande et moyenne distribution. De nombreuses autres initiatives sont portées par des associations caritatives de quartier.Le public va se diversifier dès le début de la crise économique : d'un public représenté comme étant des personnes en grande exclusion et marginalisées et à qui «on devait donner à manger », ces associations voient arriver des familles monoparentales à petits revenus, des gens en situation de chômage, des migrants en situation illégale ou en attente de régularisation, des demandeurs d'asile, des familles en attente d'instruction de leur dossier de demande d'aide financière, etc... Dans les années 90, ces associations se rendent compte que la réponse à l'urgence ne concerne pas l'ensemble de

\footnotetext{
${ }^{5}$ Article 238 bis du Code général des impôts, voté en 1988.
} 
ces demandeurs ; une partie d'entre eux cherche aussi des réponses de lien social. Des initiatives vont se développer pour tenter d'améliorer l'alimentation, de veiller à des interventions moins stigmatisantes et plus soucieuses des personnes. L'ensemble des structures publiques et privées engagées dans l'aide alimentaire va diversifier ses propositions en ajoutant des actions : les Restos du cœur et les jardins, les banques alimentaires et les différents ateliers cuisines, budgets. L'aide alimentaire ne s'inscrit plus seulement dans des interventions d'urgence mais dans des actions au long court.

\subsubsection{Les épiceries sociales et solidaires : une innovation sociale dans le panorama de} l'aide alimentaire

Dans le même pas de temps, des épiceries sociales et solidaires voient le jour comme alternative à la seule distribution et signalent un changement dans la conception de l'aide alimentaire comme objet caritatif ${ }^{6}$. En 2001, certaines de ces épiceries décident de s'organiser sous la forme associative pour avoir une représentation nationale : l'Association Nationale de Développement des Épiceries Solidaires. L'ANDES a pour objectif d'accompagner la création de projets d'épiceries solidaires et à travers les épiceries qu'elle rassemble, «de promouvoir l'accès des personnes en difficulté à une véritable citoyenneté économique : en permettant à un public exclu des circuits de consommation traditionnels de choisir les produits qu'il souhaite consommer, en vendant, à un faible prix, des denrées de qualité ; en proposant des activités où les compétences de chacun sont mises en avant, les épiceries

\footnotetext{
${ }^{6}$ La première épicerie sociale a été créée à Nevers en 1996. Elles ont d'abord été portées par des Centres Communaux d'Action Sociale puis par des associations. Dans la perspective de création de modèle alternatif on peut aussi mentionner l'initiative des Paniers de la mer créée en 1997 dans le Finistère entre trois acteurs locaux (le comité d'emploi du Pays Bigouden, la Banque alimentaire du Finistère et le Secours populaire local) dans le but de lutter contre le gaspillage en récupérant le poisson invendu, participer à la distribution de l'aide alimentaire, favoriser l'insertion professionnelle de personnes en situation de précarité et répondre aux besoins de main d'œuvre qualifiée de la filière. La création d'une Fédération des Paniers de la Mer après la naissance de la 3ème association s'est organisée autour d'une charte commune (Paturel, 2013).
} 
solidaires et sociales se veulent, au-delà d'une aide matérielle, un outil de reconstruction et un tremplin vers une réinsertion durable ${ }^{7} \gg$. Cette association parle de fracture alimentaire et fait sienne la notion de précarité alimentaire. Elle part du constat suivant : plutôt que de partir d'un choix de produits contraint par l'offre, il s'agit d'offrir la liberté du choix des produits. L'ANDESfonde sa raison d'être sur un discours et des pratiques de professionnalisationet celle-ci intègre les principes de gestion entrepreneuriale qui vont notamment la faire reconnaitre comme interlocuteur pertinent des pouvoirs publics. L'apparition des épiceries sociales et solidaires marque une rupture vis-à vis des pratiques des opérateurs historiques de l'aide alimentaire (Secours Populaire, Croix Rouge, Banques alimentaires, Restos du Cœur). Dans des espaces aménagés en libre-service, aussi proche que possible d'un magasin d'alimentation générale, elles mettent à disposition de leurs usagers des produits variés et de qualité, moyennant une faible participation financière : entre $10 \%$ et $50 \%$ du prix usuel.Elles constituent en ce sens une innovation sociale organisationnelle (Besançon et al. 2013), c'est-àdire qu'elles proposent un nouveau mode d'organisation pour l'aide alimentaire basé sur des principes inédits (choix des produits et contrepartie financière). Le service de la Direction Générale de la Cohésion Sociale en charge de l'aide alimentaire ne s'y trompe pas et soutiendra l'ANDES dans ses diverses expérimentations dont le programme Uniterres est la dernière en date.

\section{2 : Uniterres, ou la création de « circuits courts solidaires » dédiés à l'aide alimentaire}

Le projet Uniterres remet en cause le modèle classique des filières d'approvisionnement de l'aide alimentaire et crée de nouvelles relations entre acteurs dans un contexte de financement renouvelé (1.2.1). Le projet consiste à mettre en place de nouvelles filières en circuits courts,

\footnotetext{
${ }^{7} \mathrm{http}: / /$ www.epiceries-solidaires.org
} 
ce qui pose de sérieux défis en termes de financement. Son fonctionnement repose actuellement sur le Crédit National des Epiceries Sociales (CNES) pour les produits et sur un assemblage de subventions publiques et privées (fondations) pour la logistique et les charges de personnel (1.2.2 et 1.2.3). En outre ce projet pose également question en termes de stabilité des approvisionnements puisqu'il ambitionne de se fournir auprès de producteurs considérés comme fragilisés en leur fournissant à la fois un débouché mais également un appui en gestion de leur exploitation.

\subsubsection{Agriculture et aide alimentaire : du PEAD au CNES}

L'aide alimentaire a depuis $1987^{8}$ joué un rôle de "variable d'ajustement" de la surproduction agricole avec l'écoulement des stocks d'intervention issus de la régulation des marchés agricoles de la Politique Agricole Commune (PAC) dans le cadre du PEAD (Programme européen d'aide aux plus démunis)(Paturel, 2013; Duboys de Labarre et al. 2016). Mais progressivement les surplus agricoles (sucre, beurre, riz, céréales) disparaissent avec la réforme de la $\mathrm{PAC}$ et le PEAD est doté financièrement pour l'acquisition de denrées alimentaires sur le marché (via des appels d'offre dont les conditions varient selon les pays) (rapport de la cour des comptes européenne, 2009). L'inversion du rapport entre les denrées provenant de stocks d'intervention et celles achetées occasionne un litige entre les pays membres qui sera tranché par l'arrêté du tribunal européen du 13 avril 2011. La proportion des denrées achetées par rapport au volume total du plan étant passée de 18,06 \% en 2006 à $85,35 \%$ en 2008 et à 86,98 \% en 2009. A partir du 1er janvier 2014, le PEAD est remplacé par le Fonds d'aide aux plus démunis (FEAD), annexé au Fonds social européen (FSE) et non plus à la PAC. Ce faisant, les denrées doivent être mises à disposition gratuitement, ce qui va

\footnotetext{
${ }^{8}$ En réalité, l'aide alimentaire joue un rôle de variable d'ajustement pour la production agricole à l'échelle internationale depuis 1954, date à laquelle la FAO a institué cette filière.
} 
à l'encontre de l'un des principes fondateurs des épiceries sociales et solidaires - la contrepartie financière, et donne lieu à des protestations. En 2014, la création du Crédit National des Epiceries Sociales ${ }^{9}$ permet aux réseaux habilités (à savoir l'ANDES, la Fédération Françaises des Banques Alimentaires FFBA, la Croix Rouge et Imagine 84) d'acheter les marchandises destinées à approvisionner les différents circuits de distribution de l'aide alimentaire. Dès lors, c'est l'opportunité pour revoir les formes d'approvisionnement de l'aide alimentaire, notamment pour les fruits et légumes : marché international, marché local ? (Caraher et Coveney, 2016).

\subsubsection{Uniterres : Rediriger les subventions étatiques de l'aide alimentaire vers l'achat en circuits courts de proximité en créant de nouvelles filières}

L'idée initiale de la conceptrice du projet Uniterres était de créer une «grande AMAP de l'aide alimentaire $»^{10}$. Lancé de manière expérimentale en Poitou-Charentes en juin 2012, Uniterres approvisionne à la mi-2016, 74 épiceries dans quatre régions ${ }^{11}$ avec les produits précommandés auprès de 160 agriculteurs. En rupture avec la logique de filières longues (appels d'offre France Agrimer, grossistes pour les restos du cœur...)Uniterresest le seul modèle qui a,dès à présent,atteint une légitimité nationale avec une présence dans $4 / 5$ régions (l'initiative des Restos du cœur étant limitée à l'Hérault).Pour l'approvisionnement en fruits, légumes et œufs en circuits courts, qui représente en moyenne $37 \%$ du coût total d'Uniterres, les fonds proviennent de la Direction Générale de la Cohésion Sociale (Ministère de la Santé et des Affaires Sociales) à travers le CNES présenté ci-dessus. Mais outre l'achat des denrées

\footnotetext{
${ }^{9}$ Le CNES va en réalité prendre la ligne budgétaire prévue pour le Plan Alimentation Insertion ; il est crédité de 7,7 millions euros sur 7 ans (2013-2020).

${ }^{10}$ Il s'appelait d'ailleurs initialement $\mathrm{A}^{2} \mathrm{MAP}$.

${ }^{11}$ Aquitaine, Midi-Pyrénées, Bretagne et Pays de la Loire.
} 
alimentaires, il convient également de financer les charges de personnels et de logistiques du programme.

\subsubsection{Mobiliser des ressources financières publiques et privées pour financer la logistique du projet ainsi que les charges de personnels}

Pour financer les charges de personnels et de logistiques du programme (actuellement 33 personnes : chef de projet, coordinateurs logistiques, animateurs en service civique....; sans compter les moyens généraux de l'ANDES), Uniterres, tout comme de nombreuses associations du secteur de l'aide sociale, mobilise tout un panel de subventions publiques et privées, notamment les fondations. Le coût moyen d'un programme oscille entre $250000 €$ et $400000 €$ par an et par région ${ }^{12}$. Le coût varie en fonction du nombre de bénéficiaires ${ }^{13}$ dans les épiceries concernées, de la localisation géographique des épiceries solidaires et des agriculteurs participant au programme (distances à parcourir pour les coordinateurs). Les subventions publiques proviennent desconseils régionaux et départementaux, des Communautés de communes (ou métropoles), des DRAAF (Direction Régionale de l'Agriculture, de l'Alimentation et de la Forêt), des MSA (Mutualité Sociale Agricole) et des ARS (Agence Régionale de Santé). L'Etat, en plus de la subvention CNES,participe directement à travers les contrats aidés dont bénéficient la quasi-totalité des salariés du programme. Les subventions privées proviennent pour leur part du Fonds Français pour l'Alimentation et la Santé qui a permis le démarrage du projet dans le cadre de la recherche interventionnelle ECOALES, de la fondation Daniel et Nina Carasso ${ }^{14}$, de la fondation Carrefour, de la fondation Macif, de la fondation Avril ainsi quede la fondation Anber et du

\footnotetext{
${ }^{12}$ Source : Fiche technique du programme Uniterres.

${ }^{13}$ Une enveloppe de $60 €$ par personne inscrite dans une épicerie solidaire est prévue dans la limite de $15000 €$ par épicerie pour une saison Uniterres (une année).

${ }^{14}$ Du nom des fondateurs de l'entreprise Danone.
} 
GNIS $^{15}$ par le don de semences.Ces subventions sont temporaires et il faut réitérer les demandes de financement périodiquement (souvent annuellement) en justifiant des résultats via des rapports chronophages. Pour répondre à la problématique de la pérennisation du modèle financier de ce projet au-delà des fonds d'amorçage, la création du volet «Uniterres Equitable » en direction de la restauration collective publique et privée apparaît comme une piste intéressante. Il permet notamment d'amortir les coûts liés à la logistique.

\section{2 : La démocratie alimentaire à travers Uniterres}

Au-delà de ce modèle socio-économique innovant, nous souhaitons ici interroger le programme Uniterres en termes de démocratie alimentaire. Ces nouveaux circuits courts permettent-ils aux plus vulnérables de se réapproprier leur alimentation pour les uns et leur production pour les autres? Dans quelle mesure permet-il aux bénéficiaires des épiceries de manger en accord avec leurs valeurs et ce dans un contexte de budget alimentaire très contraint? Dans quelle mesure permet-il aux agriculteurs de vendre leurs produits en accord avec leurs valeurs- de retrouver une certaine maîtrise sur le devenir de leur production et sur les prix de vente de leurs produits ? Pour traiter ces questions nous présentons tout d'abord notre vision de la démocratie au sein des systèmes alimentaires (2.1) puis nous la confrontons aux résultats de nos enquêtes de terrain (2.2).

\section{1 : La démocratie alimentaire en question}

Dans cette section nous développons notre conception de la démocratie alimentaire (2.1.1) ; les initiatives concrètes qui l'illustrent (2.1.2) ainsi que les défis à relever dans le domaine particulier de l'aide alimentaire (2.1.3).

\footnotetext{
${ }^{15}$ Groupement national interprofessionel des semences et des plants
} 


\subsubsection{Démocratie, justice et citoyenneté au sein des systèmes alimentaires}

Les concepts de justice auxquels s'adossent un qualificatif (agricole, alimentaire, agrialimentaire, etc.) fleurissent mais peinent à prendre en compte le rapport social dans lequel le modèle dominant du système alimentaire mondialisé s'est construit depuis 50 ans ${ }^{16}$. Le concept de «justice alimentaire » porte principalement sur l'accessibilité à une alimentation de qualité pour les consommateurs urbains comme à Détroit ou dans le Bronx à New York (Gottlieb and Joshi, 2010 ; Paddeu, 2015) alors que le concept de justice agri-alimentaire vise à intégrer les enjeux de justice au niveau des producteurs agricoles marginalisés(Hochedez et Le Gall, 2016).Nous proposons ici de privilégier le concept de démocratie alimentaire. Pour Lang (1998), la démocratie alimentaire locale désigne des processus de gouvernance alimentaire au sein desquels des collectifs de citoyens décident de leurs choix d'alimentation et mettent en place les filières adaptées à leurs choix. Ce concept a le mérite de poser à la fois les questions de justice - à travers l'accès, la participation et le pouvoir d'agir - et celles de citoyenneté, et ce pour l'ensemble des acteurs du système alimentaire (producteurs, transformateurs, distributeurs, consommateurs...).La démocratie est un système de gouvernement qui rend compte des modes de vie et des choix des citoyens, de la façon dont ils interagissent ainsi que de leur conception à propos de l'Etat et de ses institutions.Mais c'est aussi l'évolution des formes du politique, en particulier à travers une multitude de mouvements sociaux et luttes socio-politiques à travers la planète qui redéfinissent ce qu'est la démocratie. La démocratie alimentaire conceptualisée par Tim Lang (1998)et reprise

\footnotetext{
${ }^{16}$ Nous postulons que cette explosion de « justice » est d'abord liée à un rapport compétitif entre des disciplines scientifiques qui n'ont pas l'habitude de poser la question du rapport social dans sa forme renouvelée, à savoir dans la déconstruction de la domination patriarcale, raciale et du capitalisme. En ce sens nous partageons l'analyse de cette nécessité de déconstruction de Rachel Slocum, Kirsten Valentine Cadieux et Renata Blumberg dans leur article «Solidarité, espace et race : vers des géographies de la justice alimentaire » (2015).
} 
récemment par John Coveney et Sue Booth (2015) se renouvelle ainsi, en s'inscrivant dans ces nouvelles aspirations citoyennes à des nouvelles formes du politique. Mais quel que soit l'entrée utilisée pour parler de démocratie, elle s'appuie sur un principe de justice sociale qui va permettre une articulation concrète avec la citoyenneté.

\subsubsection{De nouvelles formes de démocratie réelle s'incarnant dans la vie ordinaire à travers}

les initiatives de réappropriation du système alimentaire par les citoyens

Les food movement aux Etats-Unis, les organisations des AMAP en France à travers le Mouvement inter-AMAP ${ }^{17}$, les Groupes d'achats solidaires en Italie, la mise en place de potagers ou d'agriculture dans les manifestations de contestation (Notre Dame des Landes, les jardins d'Athènes, le potager de la place de la République, etc. ) sont autant de signes de l'expression d'une reprise en main de la part des citoyens sur les aspects de vie ordinaire comme le fait de manger.Toutefois, au-delà de la simple revendication de manger, il s'agit de reprendre la main sur les choix alimentaires en demandant des comptes sur les conditions de leur production(Renting et al. 2012). Choisir d'investir le concept de démocratie dans la période actuelle de crise sociale, économique et politique, c'est aussi renouer avec les combats et luttes politiques à travers «l'exploitation pour dénoncer les injustices produites par le capitalisme et l'impérialisme ; d'oppression, pour lutter contre les dénis de liberté propres aux régimes coloniaux ou autoritaires ; d'inégalité, pour mettre fin aux politiques de discrimination ou d'ostracisme à l'égard de minorités ; ou d'aliénation, pour s'opposer aux stratagèmes que les gouvernants et les puissants utilisent pour confisquer le pouvoir, occulter leur action ou gruger les populations »(Ogien et Laugier,2014, p. 13). La tendance en France est celle de penser que les circuits courts seraient la seule et unique réponse comme

\footnotetext{
${ }^{17}$ Le MIRAMAP.
} 
nouvelle forme de démocratie. Or, le développement d'une multitude d'initiatives du côté de la société civile montre la montée en puissance des jardins (ouvriers, familiaux, partagés etc...), la récupération des denrées alimentaires - pas uniquement par les associations caritatives d'aide alimentaire $^{18}$, et la création de nouveaux commerces comme les magasins de producteurs, les magasins bio en vrac ou encore les coopératives de consommateurs ${ }^{19}$. L'alimentation est alors propulsée comme forme politique de réappropriation par les citoyens d'une démocratie réelle s'incarnant dans la vie ordinaire. La reconnexion entre agriculture et alimentation apparaît comme un terreau particulièrement propice à la construction d'une nouvelle citoyenneté. Ces initiatives, même si elles affichent une préoccupation d'égalité d'accès, peinent toutefois à le permettre réellement aux personnes les plus pauvres ou les plus vulnérables.

\subsubsection{Démocratie et aide alimentaire}

En nous appuyant sur la définition du système alimentaire comme « la façon dont les hommes s'organisent pour produire, distribuer et consommer leur nourriture » (Malassis, 1998) et au constat de la tension entre ceux qui cherchent à reprendre la main sur leur alimentation en se préoccupant de ceux qui la produisent et ceux parce qu'ils n'ont pas les ressources économiques, ont comme horizon, l'aide alimentaire (dans un pays comme la France), la notion de démocratie alimentaire prend alors tout son sens. Et ce d'autant plus, que des agriculteurs sont en situation de précarité (dont certains bénéficient de l'aide alimentaire) et que de nombreux salariés de l'agroalimentaire sont pris dans la tourmente des dépôts de bilan

\footnotetext{
${ }^{18}$ La loi 2016-138 sur le gaspillage alimentaire ouvre de telles perspectives.

${ }^{19}$ Plusieurs projets sont actuellement en œuvre en France sur le modèle de la Park Slope Food Coop à Paris, Bordeaux, Montpellier...
} 
des entreprises de ce secteur ${ }^{20}$.Dès lors, c'est tout l'enjeu d'un programme tel qu'Uniterres que de revoir les formes d'approvisionnement- et au-delà tout le système de l'aide alimentaire, pour créer les conditions d'une initiative relevant de l'idéal de la démocratie alimentaire. Or à l'heure actuelle, la notion de justice sociale, principe-clé de la démocratie alimentaire, s'énonce à travers deux types d'argumentaire (Fraser, 1998) : un premier sur le registre de la redistribution des ressources pour contrer les inégalités et un second sur le respect des différences des personnes ou de groupes sociaux (ethniques, culturels, sexuels...) qui cherchent à contrer les normes dominantes comme obligation pour accéder à un traitement égalitaire. Ce deuxième type d'argumentaire, fortement lié à l'évolution de la société dans la transformation de la place des individus et du sujet, rend visible des revendications identitaires et pose la question de la reconnaissance sociale et politique (Honneth, 2000 ; Renault, 2003). Ces deux argumentaires s'opposent et bien souvent s'excluent réciproquement. Les partisans de la redistribution vont jusqu'à affirmer que les revendications identitaires nient les rapports de domination au profit d'une revendication du sujet ; quant aux tenants de l'argumentaire identitaire, ils mettent en avant que la seule préoccupation de redistribution ne peut pas prendre en compte les aspirations subjectives des personnes et empêchent la reconnaissance de ces groupes dans l'espace public. C'est cette tension qui est à l'œuvre dans les différents projets se revendiquant de la solidarité en direction d'une population identifiée comme précaire économiquement, à laquelle on destine une réponse par l'aide alimentaire.Le choix à travers les politiques sociales de soutenir un dispositif d'aide alimentaire a deux impacts politiques importants : le premier est de continuer à banaliser l'alimentation et de lui faire jouer le rôle de variable d'ajustement dans la gestion de

\footnotetext{
${ }^{20}$ Depuis 2006, 2358 PME et TPE de ce secteur ont déposé le bilan (environ 15\%) ; premier secteur industriel en France, sur cette même décade, il y a eu une perte de 33000 emplois (source ANIA http://www.ania.net/); COFACE (http://www.coface.fr/) donne des chiffres encore plus élevés : entre novembre 2013 et novembre 2014, 4600 entreprises liées à l'alimentation, agricoles, artisanales ou agroalimentaires, ont fermé leurs portes.
} 
lapauvreté ; la mise en scène annuelle de l'augmentation du nombre de bénéficiaires par les opérateurs de l'aide alimentaire fait partie des éléments du dispositif de contrôle social (Paturel, 2013). Le deuxième impact est à la fois de rendre invisible l'enjeu de la durabilité de l'alimentation et de considérer que la population en situation de pauvreté doit être nourri à partir du «gaspillage des autres» (grande distribution, industrie agro-alimentaire, consommateurs...); la conséquence directe est d'empêcher le débat public de façon démocratique sur l'accès à une alimentation durable pour tous. Dans ce contexte, insister sur le lien entre alimentation et production devient central pour permettre une réappropriation de chacun à l'accès à l'alimentation : les circuits courts peuvent ainsi être un moyen vers cette démocratie alimentaire par les apprentissages qu'ils permettent aux producteurs, aux artisans, aux consommateurs, aux pouvoirs publics (Chiffoleau et Paturel, 2016)

\section{2 : Uniterres et la démocratie alimentaire à travers le prisme des agriculteurs}

La mise en place d'un système alimentaire démocratique suppose l'exercice d'une citoyenneté active basée sur l'autonomie de décision et d'action des acteurs visant à rétablir les conditions de la justice sociale. Le programme Uniterres a été imaginé par une salariée de l'ANDES à partir d'un double diagnostic: une relative absence de fruits et légumes frais dans les épiceries sociales et une présence croissante d'agriculteurs parmi les bénéficiaires des épiceries. Réunir ces deux publics fragilisés aux bouts d'une même filière, médiatisée par des acteurs du travail social, constitue le cœur du projet. Ce projet répond-il pour autant aux aspirations de ces deux «publics cibles »? Dans quelle mesure sont-ils associésaux processus de décisions concernant cette filière et quelle est leur capacité d'action propre ? Nous nous intéressons ici principalement aux agriculteurs en montrant qu'ils bénéficient d'espaces d'engagement et de négociation au sein du projet mais que le lien d'égale vulnérabilité entre 
eux et les consommateurs n'est pas compris et ne peut donc être partagé comme socle de solidarité.

\subsubsection{Citoyenneté : autonomie de décision et d'action des agriculteurs impliqués dans}

\section{Uniterres}

Nous abordons ici les conditions d'exercice de la citoyenneté dans le cadre du projet Uniterres à travers la capacité d'action propre (Giddens, 1987) dont disposent les différents agriculteurs. Cette condition, mesurée à travers la construction d'un indice d'autonomie,a été croisée avec un indice de viabilité économique des exploitations. Les producteurs s'engagent librement au sein du programme Uniterres, même s'ils sont «orientés » par les prescripteurs » du monde agricole. Ils participent de plus à des réunions collectives annuelles de négociations des prix avec la chef du projet Uniterres, que nous pouvons considérer comme une reprise en main partielle sur les prix de vente de leur production ${ }^{21}$. Toutefois, l'enveloppe globale d'achat pour le programme restant fixe, la négociation sur les prix a des répercussions sur les choix de produits - et surtout sur les volumes, qui seront finalement précommandés au niveau des épiceries $^{22}$ : si les prix augmentent, il y a moins de volumes commandés.

Nous avons mesuré l'évolution d'indices d'autonomie auprès d'un échantillon basé sur les agriculteurs impliqués dans Uniterres au démarrage du projet à partir de quatre indicateurs : le réseau social, le bien être ressenti, les conditions de vies apparentes ainsi que l'accès à l'information agricole. En croisant leur indice d'autonomie avec un indice de viabilité de leur

\footnotetext{
${ }^{21}$ Dans les faits nous avons pu observé lors de ces réunions que les agriculteurs parviennent à faire entendre leurs problématiques pour ajuster à la hausse les prix des fruits et légumes commandés, et qu'en dehors de ces réunions, des réajustements de prix à la hausse sont également possibles (cas des fraises à l'été 2013 en PoitouCharentes par exemple). On constate par ailleurs un différentiel de prix selon les régions qui est lié aux prix pratiqués localement sur les marchés de plein vent et à partir des mercuriales.

${ }^{22}$ Ce qui pose notamment problème pour les produits certifiés biologiques qui sont moins commandés que les produits conventionnels lorsque l'alternative est proposée.
} 
exploitation agricole (basé notamment sur leurs dotations en capital physique, humain et financier et sur leur maîtrise des risques), on constate qu'à l'issue des deux premières saisons Uniterres, seuls 12 agriculteurs sur les 19 de notre échantillon peuvent être considérés comme dans de bonnes conditions pour exercer leur citoyenneté dans les espaces ouverts, c'est-à-dire dans la catégorie «vrais résilients «(à la fois indices d'autonomie et de viabilité de l'exploitation supérieure ou égale à la médiane du groupe) ou dans la catégorie « résilients potentiels » (indice d'autonomie supérieure à la médiane du groupe mais indice de viabilité de l'exploitation inférieur à la médiane du groupe) (voir graphique). Notre travail sur les indicateurs de résilience montre que le programme Uniterres active finalement trois leviers principaux : l'élargissement du capital social des agriculteurs, l'amélioration de leur santé morale ainsi qu'une meilleure maîtrise sur les prix et débouchés de leur production.

\section{Graphique : Esquisse typologique de la résilience des agriculteurs Uniterres entre viabilité et autonomie (Poitou-Charentes et Aquitaine)}

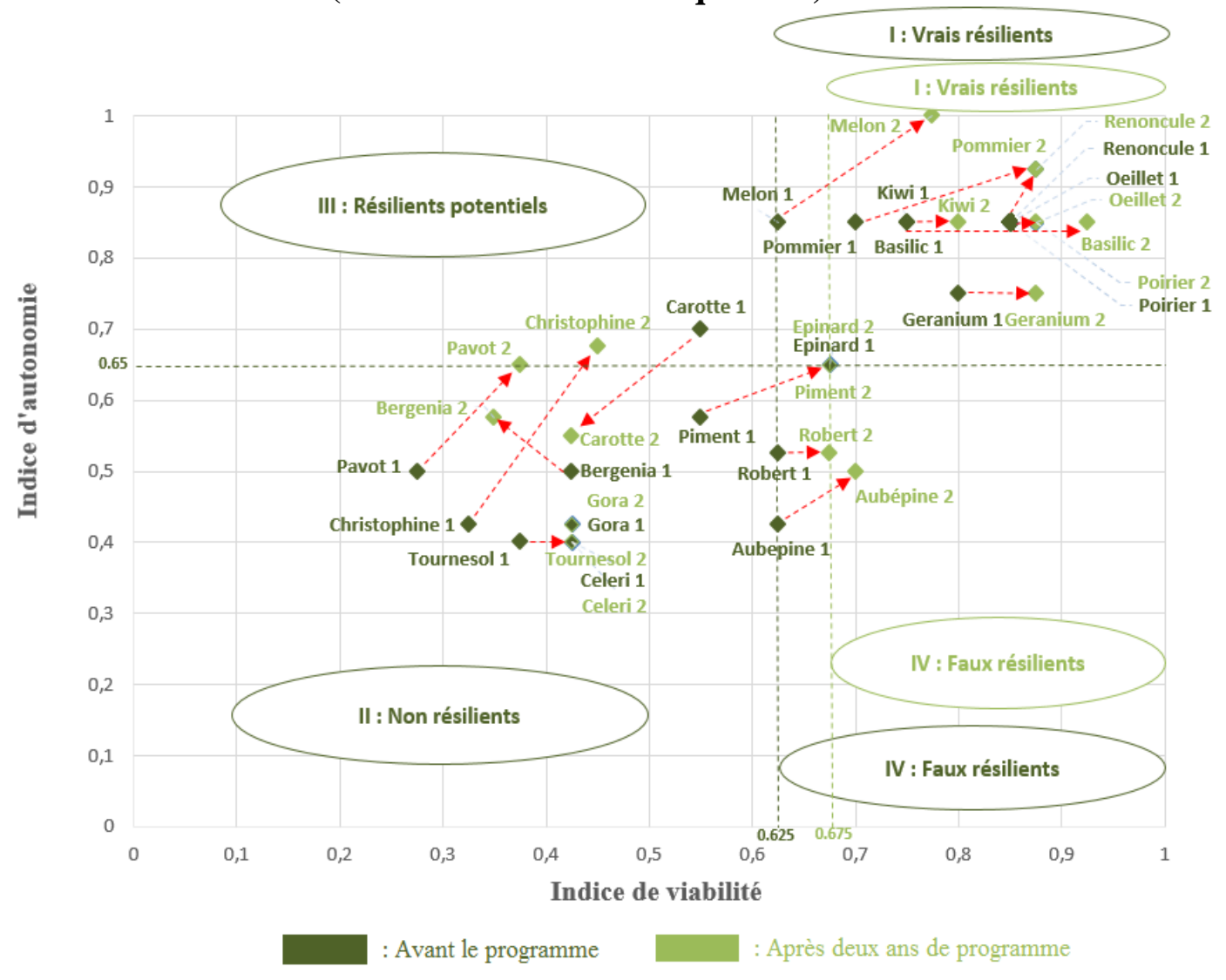




\subsubsection{Bénéficiaires des épiceries et agriculteurs «fragilisés »: des associés solidaires? La} vision des agriculteurs.

Pour les solidaristes (Bourgeois, cité par Paugam, 2008) la notion de « quasi-contrat social » est fondée sur l'idée que «la justice ne peut exister entre les hommes que s'ils deviennent des associés solidaires en neutralisant entre eux les risques auxquels ils sont confrontés ». Cette neutralisation des risques n'existe pas réellement au sein d'Uniterres car si les agriculteurs ne produisent pas, ils ne sont pas payés (à la différence des Amaps par exemple) ; cependant, nous avons vu comment Uniterres sollicite des soutiens à la fois dans son réseau d'acteurs (notamment les autres agriculteurs) et auprès de la Mutualité Sociale Agricole lorsque l'un des agriculteurs est en difficulté.Penser la solidarité entre les producteurs, et pas uniquement de l'ANDES vers les producteurs et des producteurs vers les bénéficiaires des épiceries pourrait en ce sens constituer une piste d'évolution du programme.La majorité des agriculteurs impliqués dans Uniterres se perçoivent plus dans une relation d'aide envers les bénéficiaires des épiceries que dans une relation réciproque d'entraide comme l'illustrent ces propos tenus par des agriculteurs du programme: «J'aime bien l'aspect social aussi, c'est pour ça que j'y suis », «Pouvoir donner des bons légumes à une population qui n’y a pas souvent accès »....La plupart des agriculteurs enquêtés n'ont pas d'échanges avec des bénéficiaires de l'aide alimentaire. Or la compréhension des difficultés rencontrées par les bénéficiaires, notamment sur le plan de l'alimentation, ne peut se faire par des interactions concrètes dans lesquelles la vulnérabilité de ces personnes s'exprime par des mots, des attitudes, et se matérialise sous la forme de signes, d'objets. L'attention qu'ils portent au problème d'accès à l'alimentation que rencontrent les usagers des épiceries solidaires se fait par l'intermédiaire d'expériences subjectives de précarité, passées ou actuelles, vécues par les 
agriculteurs à la première ou la troisième personne. Les récits des agriculteurs à propos de leurs parcours et situations socio-professionnelles comportent diverses descriptions d'évènements (licenciements, démissions, séparations conjugales, crises économiques de l'activité agricole parentale etc.), qui affectent ou ont affecté, de manière temporaire ou longue, leurs capacités ou celles de leurs proches à répondre à leurs besoins fondamentaux : se nourrir, se vêtir, se loger. L'engagement solidaire des agriculteurs dans le programme Uniterres puise ici sa source dans des situations de vulnérabilité dont ils sont témoins, mais surtout sujets.C'est par l'intermédiaire de leurs propres expériences subjectives de la fragilité que les agriculteurs accèdent, sur un mode réflexif, à la vulnérabilité des usagers des épiceries solidaires. La plupart des agriculteurs enquêtés soulignent que les difficultés des usagers des épiceries solidaires leur sont étrangères, dans le sens où ils n'ont pas une connaissance claire et précise de leurs difficultés. Cependant, dans le même temps, ils adoptent une posture compréhensive, voire empathique à l'égard des bénéficiaires de l'aide alimentaire l'A.N.D.E.S, en s'appuyant sur leurs propres expériences de la fragilité.D'autre part, tous se perçoivent comme producteur à qui Uniterres achètent leurs fruits, légumes et œufs ; mais aucun d'entre eux ne se perçoit comme subventionné par les crédits de l'aide alimentaire alors que c'est pourtant la subvention du Crédit national des épiceries sociales, partie prenante du financement public du dispositif d'aide alimentaire français, qui sert à ces achats. Le lien d'égale vulnérabilité entre eux n'est pas compris et du coup, ne peut être partagé comme socle de solidarité. Si des espaces de rencontres entre les consommateurs et les producteurs ont été imaginés, à travers les «tables de producteurs ${ }^{23}$ » et les visites d'exploitation, ils sont «encadrés » par des salariés et des bénévoles de l'ANDES, qui peinent à mobiliser les bénéficiaires sur ces actions. Le potentiel de création de lien social entre ces deux maillons

\footnotetext{
${ }^{23}$ Un chef cuisinier est invité pour un atelier-cuisine avec les bénéficiaires des épiceries à partir des produits des producteurs Uniterres.
} 
est donc limité et dans tous les cas, médiatisé par des professionnels du travail social.Les producteurs qui ont participé à ces dispositifs d'animation mettant en contact les bénéficiaires, les bénévoles et les salariés des épiceries sociales avec eux ramènent une vision incarnée de cette solidarité; cependant, ils sont encore peu nombreux à avoir vécu ces espaces d'intermédiations entre monde de l'action sociale et monde agricole et surtout, l'expérience montre qu'il ne s'agit pas de le mettre en œuvre une seule fois pour qu'il y ait transformation sociale.

\section{Conclusion}

Nous avons montré à travers cette communication que les acteurs de l'aide alimentaire sont confrontés à de nouvelles problématiques dans un contexte de relocalisation de leurs filières d'approvisionnement. Pour la mise en place de circuits courts solidaires, les salariés de l'ANDES ont notamment dû élargir leurs connaissances sur la précarité au monde agricole et notamment à la situation de la petite agriculture locale (maraîchage principalement). En termes de démocratie alimentaire, nous avons postulé que pour atteindre cet idéal basé sur l'exercice de la citoyenneté et la poursuite d'une meilleure justice sociale, les initiatives telles Uniterres étaient face au défi d'atteindre un double équilibre d'autonomie d'action et décision à la fois du côté des producteurs et des consommateurs. Si les agriculteurs s'engagent dans le programme Uniterres afin de bénéficier d'un appui économique pour améliorer la viabilité de leurs activités agricoles, celui-ci ne réside pas seulement dans la valeur économique des quantités de biens alimentaires qu'ils vendent aux épiceries solidaires. Il réside également dans une économie de temps et d'argent, la collecte et la livraison des commandes étant 
réalisée par les coordinateurs du programme Uniterres.Cependant la solidaritée ${ }^{24}$, affichée comme étant au cœur du dispositif a encore du mal à circuler entre les différents groupes d'acteurs : les producteurs et les bénéficiaires à chaque bout de la chaîne, les épiceries avec leurs salariés, leurs bénévoles et les prescripteurs de l'aide alimentaire, les salariés du programme Uniterres et leur liens avec l'association ANDES porteuse du programme. L'élaboration participative - avec les bénéficiaires et les producteurs - d'une charte solidaire, leur permettrait par exemple de mieux s'approprier le caractère réciprocitaire du programme. Se pose donc la question plus large de modalités démocratiques permettant à ces différents groupes d'acteurs de co-construire la dimension solidaire de ce programme.Le contexte de la société française en particulier mais des pays du Nord en général, ne permet pas l'existence d'un débat public démocratique sur l'accès à une alimentation durable pour tous, et l'exemple de l'aide alimentaire montre bien les processus d'invisibilisation des conditions de l'accès, tant du côté des bénéficiaires, des producteurs et des acteurs de l'intermédiation sociale.

\section{Bibliographie}

Agreste Primeur (2012), «Commercialisation des produits agricoles », [en ligne] http://agreste.agriculture.gouv.fr/IMG/pdf_primeur275-2.pdf

\section{Angiotti}

Bellin-Lestienne C., Deschamps V., Noukpoapé A., Hercberg S., Castetbon K. (2007), "Alimentation et état nutritionnel des bénéficiaires de l'aide alimentaire », Étude Abena, 2004-2005, Institut de veille sanitaire.[en ligne]

http://opac.invs.sante.fr/doc_num.php?explnum_id=3500

Besançon E., Chochoy N., Guyon T. (2013), L'innovation sociale. Principes et fondements, Editions L'Harmattan, Paris.

Booth S., Coveney J. (2015),Food democracy. From consumer to food citizen, Springer, Londres.

\footnotetext{
${ }^{24}$ Dans cet article, rappelons que notre focus est sur les producteurs..
} 
Caraher M., Coveney J.(2016),Food poverty and insecurity : international food inequalities, Springer, Londres.

Chiffoleau Y. (2012), « Circuits courts alimentaires, dynamiques relationnelles et lutte contre 1'exclusion en agriculture », Économie rurale, n³32, pp. 88-101.

Chiffoleau Y., Paturel D. (2016), «Les circuits courts alimentaires pour tous, outils d'analyse des innovations sociales », in Innovations $\mathrm{n}^{\circ} 50$, pp 191-210

Deverre N. et Lamine C. (2010), Les systèmes agroalimentaires alternatifs. Une revue des travaux anglophones en sciences sociales, Economie rurale $\mathrm{n}^{\circ} 317$, p 57-73

Duboys De Labarre M., Carimentrand A., Crenn C., Delavigne A.-H., Montagne K., Parvu C., Paturel D., Précigout F., Techoueyres I. (2016), "Creating new links between agriculture and food aid: new perspectives from France », in Food poverty and insecurity: the poor in a world of global austerity, coordonnée par M. Caraher et J. Coveney, Springer International Publishing, p.67-77.

Fraser N. (1998), «Penser la justice sociale : entre redistribution et revendications identitaires », in Politiques et Sociétés n³, pp 9-36

Giddens A. (1987), La constitution de la société. Éléments de la théorie de la structuration, PUF, Paris.

Gottlieb R., Joshi A. (2010), Food justice. Food, health and the environment, MIT Press, Cambridge.

Hassanein N. (2003),"Practicing food democracy: a pragmatic politics of transformation", Journal of rural Studies, volume 19/1, pp. 77-86

Hochedez C., Le Gall C. (2016), «Justice alimentaire et agriculture », justice spatiale | spatial justice, $n^{\circ}$, Janvier 2016, [en ligne] http://www.jssj.org/

Honneth A. (2000), La lutte pour la reconnaissance, Paris, Éditions du Cerf.

Hrabanski, M (2011), «Souveraineté alimentaire. Mobilisations collectives agricoles et instrumentalisations multiples d'un concept transnational », Revue Tiers Monden ${ }^{\circ} 207$, pp. 151-168

Lamine C., Perrot N. (2008) «Les AMAP: un nouveau pacte entre producteurs et consommateurs? », Yves Michel, Paris.

Lang T. (1999)," Food policy for the 21st century: can it be both radical and reasonable?", In M. Koc, R. MacRae, L.J.A. Mougeot, J. Welsh (Eds.), For Hunger-proof Cities: Sustainable Urban Food Systems (1999), International Development Research Centre, Ottawa, pp. 216224

Malassis L. (1994), Nourrir les hommes, Dominos-Flammarion, Paris 
Observatoire Economique et Social MSA (2015), «Le niveau de vie des ménages bénéficiaires des prestations de la branche Famille ou du minimum vieillesse au régime agricole en $2013 »$.

Ogien A., Laugier S., (2014),Le principe démocratie: enquête sur les nouvelles formes du politique, La Découverte, Paris.

Paddeu F. (2015), «De la crise urbaine à la réappropriation du territoire : Mobilisations civiques pour la justice environnementale et alimentaire dans les quartiers défavorisés de Detroit et du Bronx à New York », Thèse de doctorat en géographie, Université ParisSorbonne.

Paturel D. (2013), «Aide alimentaire et accès à l'alimentation » [en ligne]http://www.academia.edu/19835962/Aide alimentaire et accès à lalimentation en France

Paturel D., Marajo-Petitzon E., Chiffoleau Y. (2015), La précarité alimentaire des agriculteurs », in revue Pour, $\mathrm{n}^{\circ} 225$, pp 77-81.

Paugam S. (1991), La disqualificationsociale, PUF, Paris.

Paugam S. (2008), Le lien social, collection « Que sais-je ? », PUF, Paris.

Renault E. (2000),Le mépris social: Ethique et politique de la reconnaissance, Editions du Passant.

Renting H., Schermer M., Rossi A. (2012), "Building food democracy :exploring civic food networks and newly emerging forms of food citizenship", in International Journal of sociology of agriculture and food, $\mathrm{Vol} .19 \mathrm{n}^{\circ} 3, \mathrm{p} .293$

Slocum R., Cadieux K. V., Blumberg R. (2015) « Solidarité, espace et «race» : vers des géographies de la justice alimentaire »,Justice spatiale | Spatial justice, n ${ }^{9}$, Janvier 2016, [en ligne] http://www.jssj.org 